\title{
En Grèce antique, la douloureuse obligation de la maternité
}

Lydie Bodiou, Pierre Brulé et Laurence PIERINI

\section{(2) OpenEdition \\ 1 Journals}

\section{Édition électronique}

URL : https://journals.openedition.org/clio/1441

DOI : 10.4000/clio.1441

ISSN : 1777-5299

Éditeur

Belin

\section{Édition imprimée}

Date de publication : 1 avril 2005

Pagination : 17-42

ISBN : 2-85816-781-8

ISSN : $1252-7017$

Référence électronique

Lydie Bodiou, Pierre Brulé et Laurence PIERINI, « En Grèce antique, la douloureuse obligation de la maternité », Clio. Histoire, femmes et sociétés [En ligne], 21 | 2005, mis en ligne le 01 juin 2007, consulté le 22 avril 2022. URL : http://journals.openedition.org/clio/1441 ; DOI : https://doi.org/10.4000/clio. 1441

Ce document a été généré automatiquement le 22 avril 2022.

Tous droits réservés 


\title{
En Grèce antique, la douloureuse obligation de la maternité
}

\author{
Lydie Bodiou, Pierre Brulé et Laurence PIERINI
}

1 Qu'elle est difficile à saisir la maternité en ces cultures d'omnipotence paternelle. D'abord, en raison même de cette hégémonie, bien sûr, mais aussi - techniquement - en raison des conséquences qu'elle ne manque pas d'avoir sur les témoignages, leur origine, leur nature, leur dispersion. On n'hésitera donc pas à introduire la figure évanescente de la mère en Grèce antique par une accumulation de contradictions au regard de la méthode historique. Tentant d'en circonscrire l'image, on se heurte en effet à cette contradiction de devoir emprunter la quasi-totalité des sources primaires à la littérature de fiction, et spécialement à la tragédie, comme si elles disaient le réel. Ceci, pour deux raisons concourantes. D'un côté, un significatif constat de carence : exception faite des laconiques épitaphes, les mères sont absentes des sources textuelles; ni les historiens, ni les philosophes et autres n'ont porté attention à l'aventure spécifique de la maternité : tout ce qu'on en pourrait dire, c'est qu'ils l'ont «politiquement » interprétée. De l'autre, l'abondance : les mères emplissent le volume sonore du théatre tragique grec de leurs cris, des cris de douleur. Dans la mesure où l'espace est mesuré et où nous préférons éclairer un aspect de la question plutôt que de succomber à la tentation du tout dire, il ne reste donc plus, sur ce chemin, que d'aller à la rencontre de celui des trois Tragiques qui les a le plus souvent mises en scène: Euripide ${ }^{1}$. Aussi sera-ce principalement la voix de ses héroïnes qui viendra interpréter et il ne faut jamais oublier que c'est face aux hommes de la cité assemblés au théâtre l'immense douleur des mères grecques. Car c'est bien cette douleur que nous cherchons à rendre audible. Allant successivement d'une pièce singulière - l'Ion - à l'ensemble de son œuvre, et à la recherche d'une pensée commune sur la maternité, nous savons n'y recueillir qu'un écho (par rapport au réel comme par rapport à ses diverses traductions mentales) de ce que cette aventure signifiait dans la biographie féminine

Créuse fille, violée, mère et orpheline de fils, épouse sans enfant...

2 Erechthée est à Athènes, il est roi sans héritier mâle. Il a donné sa fille unique Créuse en mariage à un étranger, Xouthos. Par la volonté de son beau-père, celui-ci devient ainsi 
roi d'Athènes (sans autre raison que sa vaillance). Mais le malheur poursuit la maison d'Athènes, car ce mariage aussi reste infécond. Après des années d'espérance, le couple se rend donc à Delphes afin d'y consulter Apollon à propos de sa stérilité.

Les maternités tragiques de l'Ion d'Euripide

3 Cette histoire d'impasse dynastique se double d'un malheur antérieur. Quand la princesse était encore parthénos (fille vierge) au palais de son père (sur l'Acropole), Apollon l'avait violée. "À l'insu de son père - car le dieu le voulait - elle porta son fruit $»^{2}$. Elle exposa le bébé "dans le cercle parfait d'une creuse corbeille». Comme toujours dans ces divines histoires, le nouveau-né ne meurt pas. Son père Apollon le fait recueillir par son frère Hermès et transporter à Delphes. C'est là que quelques années plus tard, l'action le retrouve, serviteur de la Pythie. Erechthée ni Xouthos ne savent rien de tout cela. Et quand le néo-roi s'adresse à l'oracle apollinien, celui-ci a beau jeu de répondre malignement qu'il a un héritier, sans le savoir. Lequel ? Le premier qu'il rencontrera : c'est Ion! Déjà dans la pièce, le jeune homme avait confié son malheur d'être orphelin; Xouthos s'emploie à le persuader - difficilement - qu'il est bien son père. Mais qui est sa mère et n'a-t-elle pas fauté ?

Créuse, seule avec les spectateurs à savoir qu'elle est féconde, mère d'un enfant qu'elle croit mort, apprend que son mari, infécond en leur couple, est désormais pourvu d'un fils. Humiliée et furieuse, elle décide de faire empoisonner ce fils, dont elle ignore qu'il est le sien! Démasquée, elle se réfugie en suppliante à l'autel d'Apollon où la Pythie lui remet cette fameuse corbeille où elle avait abandonné l'enfant. Son fils la trouve là. Il se rend aux preuves matérielles de sa filiation maternelle. Le voilà maintenant pourvu d'une mère sans reproche. Athéna clôt le drame en expliquant comment Apollon a "donné » son fils à Xouthos "pour lui assurer un patrimoine ». Créuse, sauvée de l'infanticide retrouve sa fécondité.

5 Si l'on réduit l'action à son canevas dramatique essentiel, il s'agit de transformer le fils biologique d'un dieu, né du viol d'une princesse vierge en un fils social et politique d'un mari stérile. À la clef, et en vertu de cette "adoption" par le père social, le trône d'Athènes ira à ce fils «fabriqué $»^{3}$. C'est la volonté du dieu. Et cette introduction d'Ion dans la lignée royale d'Athènes rend possible la raison pour laquelle tout cela est imaginé. En effet, à la différence de ce qui se passe dans nombre de cités, le dieu ancestral des Athéniens, celui des familles et des groupements de parenté symbolique, ce n'est pas Zeus - appelé dans ce contexte, Zeus Patrôos -, mais Apollon - Apollon Patrôos ${ }^{4}$. Le scénario de l'Ion répond à cette double nécessité d'expliquer 1 - l'éponymie des Ioniens (ce rameau du peuple grec, au premier rang desquels se placent les Athéniens eux-mêmes; Ion > Ionie) : ils descendent de cet ancêtre, fils d'une princesse athénienne ; 2 - la raison pour laquelle cet Apollon est leur dieu « ancestral».

6 Cette histoire met rudement à l'épreuve les concepts de paternité et de maternité Successivement, et parfois simultanément, Créuse vit trois rapports très contrastés à la maternité. D'abord le viol, et une maternité subie, dramatique. Dans le temps même de cette révélation, la pression sociale de l'honneur l'oblige à y renoncer, la contraignant à exposer l'enfant. Puis, alors qu'on lui a donné Xouthos pour époux, vient la longue et douloureuse période de maternité orpheline : à la fois sa recherche solitaire et cachée de cet enfant entrevu, celui dont elle ignore qu'il a survécu à Delphes, et, dans le même temps, son attente déçue d'une maternité nouvelle, avec cette douleur quand son corps ne répond pas aux attentes de descendance du couple, thème qui prend le plus de place dans la pièce. Et voilà qu'à Delphes, elle retrouve son fils. Elle jouit enfin de cette 
maternité joyeuse, maternité sans grossesse, qu'elle-même et le chœur plaçaient toujours en opposition avec son déchirement, et que fait surgir ce coup de théâtre. Et puisqu'il faut un happy end, l'accord désormais scellé entre Apollon, Ion et Créuse fait de Xouthos le père d'un fils dont tout le monde sauf lui sait qu'il n'est pas le sien, mais réinstalle aussi Créuse comme mère sociale après qu'eut été reconnue sa maternité biologique. Enfin hors de la scène tragique, et comme si l'infanticide avait jusqu'alors bloqué sa fécondabilité, elle donnera d'autres princes à sa cité.

7 Dans l'ordre du discours, il en va de la maternité comme de la pureté : il est inverse. De la même façon que c'est par le discours sur la souillure qu'on en apprend le plus sur cette dernière, si l'Ion parle bien de la mère et de la maternité, c'est en évoquant l'absence de descendance, la stérilité de la femme. Les termes qui reviennent le plus fréquemment sont ceux qui disent ces vacuités, les mots de la famille d'apaidia et d'ateknia. Ce que l'Ion a de particulier, c'est que ces mots sont indifféremment appliqués au couple, à l'épouse et au mari.

Chercher le père et chercher la mère

8 Ce nourrisson exposé par Créuse, Apollon voulut, « chez lui, l'avoir pour serviteur " ${ }^{5}$, la Pythie le recueillit, «l'éleva, sans savoir qui était son père, ni quelle mère l'avait conçu $»^{6}$. Apollon a bien ensemencé Créuse, Ion a bien un géniteur, et c'est bien une mèter ('mère') qui l'a fait (ephu). Ce verbe phuein étonne ici, habituellement dévolu plutôt au rôle masculin dans la génération. Cela sonne comme si elle l'avait fait toute seule. C'est sans doute la singulière origine du garçon qui motive ce transfert. Un enfant, né de l'improbable union d'un dieu et d'une parthénos ('vierge'), c'est un parthénios (un "virginal»), un sans 'maison', un sans nom ${ }^{7}$, un rien du tout social, auquel il ne reste que la vie et sa piété envers Apollon. L'amour d'un fils, normalement, va à son père, celui auquel il doit son germe ${ }^{8}$. Mais lui, son père lui manque. C'est que, dans le temps d'Euripide, et du côté du père, la distinction entre le rôle génésique et le rôle social n'est pas oiseuse. En pays grec, la fréquence inusitée de l'adoption et sa valeur symbolique contribuent à disjoindre clairement les deux rôles. Par ailleurs, naître d'un père ne suffit pas pour en hériter ni pour lui succéder. Pour faire d'un fils biologique son fils social et politique il convient que le père ajoute à son engendrement des rites et des démarches qui accompagnent la croissance de son garçon. Ce sont là obligation et privilège du masculin, la mère évidemment n'en jouit pas, qui se contente de fabriquer et de maintenir en vie. Quant à Créuse, tout un chacun la croit vierge (mais non les spectateurs), et elle est une fille sans frère ${ }^{9}$, de ces filles que tout père grec «normal» se préoccupe de marier dignement, dans la perspective de se procurer l'héritier qui manque à sa lignée - ici, celle, insigne, des Erechthéides. Se présente à l'esprit du roi le parti de Xouthos, un étranger certes, mais qui n'est pas sans qualité puisque, dans une guerre contre les Chalcodontides d'Eubée, il fut son allié victorieux. Érechthée, se conformant à la structure du mariage en gendre ${ }^{10}$, lui offre donc sa fille « en dot de guerre $»^{11}$, prix de sa vaillance. Las! Cet héritier qu'il recherche au travers du gendre, ce petit-fils qui est l'obsession des pères grecs, sa fille ne le lui donnera pas. «Il y a longtemps que [Xouthos] ensemence la couche (speiras lechè) de Créuse, mais il est sans enfants » (ateknos) $)^{12}$.

9 Or, les spectateurs, avertis par Hermès depuis le début du prologue, savent que Créuse est féconde, que le sperme violent du dieu l'a engrossée. Ils savent donc aussi qui est responsable de la stérilité de son couple, que Créuse n'y est pour rien et que cette anomalie incombe à Xouthos. On ne sait pas trop si Créuse aime Xouthos, ni s'il l'aime, 
cela n'intéresse pas Euripide (i.e. les Athéniens). Ce que l'on sait, c'est qu'ils souffrent tous les deux de ne pas remplir cette obligation sociale - et aussi politique - qui consiste à faire des enfants - c'est-à-dire un fils. Or, cette calamiteuse agénésie est normalement, habituellement, considérée comme d'origine seulement féminine. C'est parce que tout un chacun pense ainsi que Créuse est venue aussi à Delphes. Si, par extraordinaire, Xouthos avait été désigné comme le responsable, il serait venu seul pour consulter le dieu. Mais cette tare de l'ateknia est une malédiction de femme. Les spectateurs, eux, savent que les semences de Phoibos et de Xouthos sont sans commune mesure, et Créuse le sait, mais elle ne saurait le dire. Alors la place de Xouthos est assez ridicule, elle ressemble à celle du mari trompé (ce qu'il est d'une certaine façon) : tout le monde connaît son infortune, lui l'ignore. Le mutisme quasi-religieux, en tout cas tout de révérence de Créuse envers son divin violeur, la fait épouser le rôle que lui reconnaît l'opinion commune, elle qui fait de la femme le fauteur de trouble dans le processus reproductif et lui fait porter l'essentiel du fardeau de l'infertilité. Mais, dans ce partage des rôles, il faut se garder de trop simplifier; ils sont deux sans enfant et même dans l'échange avec son fils, Créuse partage cette situation avec son mari :

Créuse. - Notre longue union est demeurée stérile (apaidia).

Ion. - Jamais tu n'as mis au monde aucun enfant (ateknos)?

Créuse. - Hélas! Phoibos sait bien comment j'en suis privée (apaidia) ${ }^{13}$.

Voyez les mots. Quelle insistance sur cette stérilité ! Et, cette image de la femme apais, inutile voire nuisible, se construit en opposition avec celle de la femme fertile en enfant, la femme eupais. Tout est dans cette différence qui suffit à tout inverser :

Ion. - Pauvre femme, heureuse en tout le reste, mais bien malheureuse !

11 Créuse ne répond pas, mais par une question, qui fait aussi progresser l'intrigue, elle interroge ainsi Ion sur sa mère :

Créuse. - Et toi, qui es-tu ? Ta mère à toi est bien heureuse ! ${ }^{14}$

12 On traduirait plus exactement: celle qui t'a enfanté (tekousa) connaît la félicité. Inversement, selon le chœur, il ne reste à celles qui n'en peuvent mais, qui ne portent pas d'enfant, à celles qui voient arriver dans leur maison un enfant " apporté » par le mari, il ne leur reste qu'à « vieillir, grisonnante ", à se ratatiner au fond des demeures, et, même si le couple est sans orage, à être privée des tendres témoignages de l'époux ${ }^{15}$. Le désir est mort. C'est bien ainsi en effet que tout au long de la pièce, tous les protagonistes à leur tour, et aussi le chœur formé des servantes de Créuse, proclament en creux la félicité que procure la maternité. C'est ce que met en lumière par contraste cet aveu du chœur à Créuse quand elle vient demander quel oracle est venu du dieu pour la guérir de son apaidia :

Eh bien, je parlerai, dussé-je mourir deux fois ! C'en est fait, maitresse : tenir un enfant dans tes bras, lui donner le sein, toi ... jamais !16

La mère, une femme accomplie

13 Créuse et Ion ignoraient le lien biologique existant entre eux, mais, par le coup de théâtre de la reconnaissance, les voilà réunis à la fin de la pièce. Créuse, privée jusqu'alors d'enfant, gagne un fils. Dans le texte tragique, les mots sont signifiants: pour traduire la transformation finale de Créuse de femme stérile en mère, le vocabulaire grec dispose de termes spécifiques qualifiant les deux états successifs de cette femme. D'apais, littéralement "sans enfant", elle devient eupais, " heureuse en enfant». Dans la classification des personnages féminins qu'utilise Euripide dans l'ensemble de son œuvre, ce changement d'état que connaît Créuse la fait passer d'une 
catégorie de femmes à une autre. Celles-ci s'y répartissent en effet rigoureusement en deux types: il y a celles qui sont mères et celles qui ne le sont pas. Les femmes appartiennent forcément à l'une ou à l'autre des deux catégories. Ce qui articule cette classification c'est la maternité, qualité que possèdent ou non les femmes. La perdre les exclut de la première catégorie et les enferme dans celle des non-mères.

Eupais versus apais

Trois types de femmes sont qualifiés dans l'œuvre d'apaides, de femmes «sans enfants ». Il s'agit de toutes celles qui ne sont pas mères, c'est-à-dire à la fois celles qui ne le seront jamais, celles qui ne le sont pas encore et celles qui ne le sont plus. Au premier rang d'entre elles arrivent les femmes stériles, car l'apaidia c'est avant tout la stérilité. L'Andromaque, par exemple, est le drame de la confrontation entre Hermione,

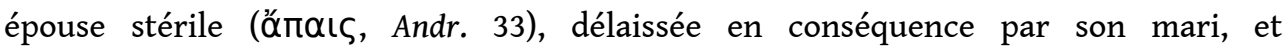
Andromaque, esclave mais féconde, mère d'un enfant qu'elle a conçu de son maître. Les mères qui ont perdu leur enfant sont également dites apaides. Ainsi, pour qualifier une Créuse, une mère rendue "orpheline" par la perte de son enfant, situation que le français ne sait pas dire en un simple mot, le grec ancien utilise le terme apais qui, dans d'autres contextes, désigne les femmes stériles. Hécube reçoit ce qualificatif, elle qui représente, dans l'œuvre d'Euripide, le «paradigme de la maternité endeuillée ${ }^{17} »$ :

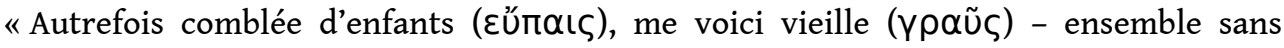

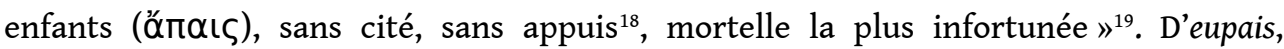
Hécube n'est plus qu'apais et ce changement fait brutalement d'elle une vieille femme. Les autres mères de l'œuvre que le drame prive de leur enfant sont également qualifiées de "vieilles femmes $»^{20}$. L'équation entre perte de l'enfant et vieillesse prématurée est systématiquement établie par le poète; elle est la clef permettant de comprendre pourquoi, dans la langue grecque, un même terme est employé à la fois pour désigner les femmes stériles et les mères frappées par la mort d'un enfant. « La mère endeuillée est toujours, chez Euripide, une mère sans espoir de maternité future, une mère qui a dépassé le stade de la reproduction, une mère "vieille" " ${ }^{21}$. La vieillesse n'est physique qu'en raison du codage scénique, afin que, visuellement, on puisse reconnaître sur scène celle qui, socialement, est « vieille » désormais. Quel que soit son âge, elle est considérée comme les femmes âgées et ménopausées qui ont dépassé le temps de la fécondité utile à la sociétée ${ }^{22}$. La mère endeuillée est alors dite apais. Elle est ainsi qualifiée du même titre que les femmes stériles qui ne peuvent donner un héritier à leur époux et à leur cité. Mais à la différence des femmes stériles qui n'ont jamais eu d'enfants, les mères endeuillées sont des "mères sans enfants " et l'oxymore que contient cet énoncé dit le caractère tragique de leur situation. De là à affirmer qu'aux yeux de la société elles ne sont plus mères, il n'y a qu'un pas que franchit finalement le

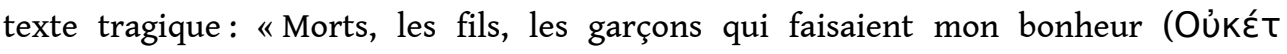

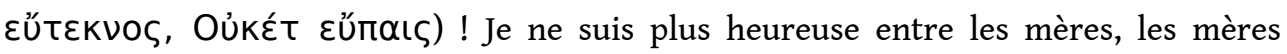
fécondes d'Argos, et l'Artémis des accouchées ne regardera plus celles qui sont sans fils

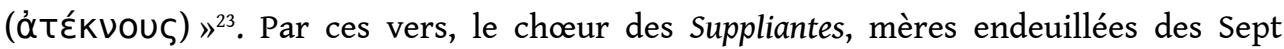
Chefs tombés au combat, traduit son exclusion de la communauté des mères. La mère apais n'est plus sous la tutelle de la divinité protectrice des couches et rejoint ainsi les femmes stériles qui n'ont pas le droit à cette protection divine. La mère apais est une mère déchue, qui a perdu son statut social de mère et qui, par conséquent, rejoint la catégorie des femmes qui ne sont pas mères. 
15 À cet ensemble féminin appartient également le chœur des Électre, Iphigénie et Macarie, toutes ces parthenoi que le drame condamne à rester vierges, donc apaides, jusqu'à la fin de leur vie. Électre constate que c'est à une mort sociale que Clytemnestre l'a condamnée : "Ma vie à moi s'en est allée tout entière dans les gémissements, les

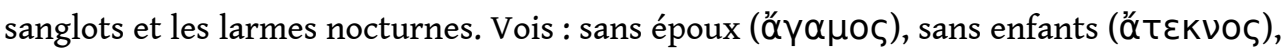
la déplorable existence que je traîne éternellement ${ }^{24}$. Elle ne connaît pas l'accomplissement féminin qui passe par le mariage et la maternité. Dans son cas, l'âge transitoire de la parthénos, qui, ayant quitté l'enfance, se prépare à devenir épouse et mère, prend un caractère permanent. Électre et les autres jeunes vierges de l'œuvre demeurent parthenoi à jamais.

16 Femmes stériles, mères privées de leur enfant et parthenoi ont un rapport différent à la maternité. Elles sont pourtant toutes qualifiées du même terme, apais, qui les exclut catégoriquement du génos des mères, de leur espèce ${ }^{25}$. Leur identité sociale n'est définie que négativement, en opposition avec les mères. Souvent, dans leurs plaintes devant le malheur qui les accable, les mères endeuillées retracent le chemin qui les a conduites de la maternité à la privation d'enfant. Hécube ou les Suppliantes rappellent que d'eupaides, elles ont été brutalement réduites à la condition d'apaides. Créuse, au contraire, en retrouvant Ion, parcourt l'itinéraire inverse : «Je ne suis plus sans fils, je ne suis plus la mère sans enfant ${ }^{26}$.

17 Le terme eupais, par son préfixe, exprime la réussite et le bonheur que représente la naissance d'un enfant pour une mère. C'est le souhait du chœur des servantes de Créuse: "Ah! Puissé-je n'avoir qu'une aisance modeste, mais, en revanche, être

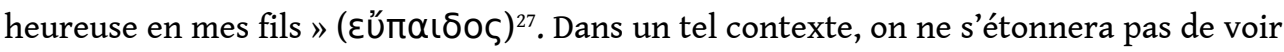
le terme eupais s'enrichir d'une nuance de fierté ; cela apparait par exemple dans le vœu des jeunes Phéniciennes qui admirent le sacrifice volontaire de Ménécée : «Ah!

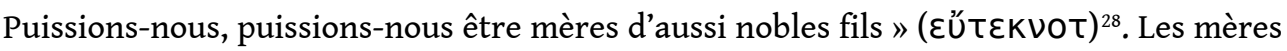
grecques mettent au monde et élèvent des enfants dans le but qu'ils servent la cité. C'est ce qu'Iphigénie rappelle à sa mère qui refuse de laisser sacrifier sa fille pour permettre le départ de la flotte grecque : "C'est pour l'ensemble de la Grèce que tu m'as enfantée, non pour toi seule $»^{29}$. Mais voilà tout le paradoxe des exigences qui sont faites aux femmes grecques. En cédant leur enfant pour le salut de la patrie, elles peuvent se considérer comme des mères eupaides et glorieuses; mais dans un même mouvement, privées de leur enfant, elles deviennent des femmes apaides qui ne peuvent plus prétendre appartenir au cercle des mères. Hécube, après la mort volontaire de sa fille Polyxène, est à la fois «la plus heureuse des mères et la plus infortunée des femmes $\|^{30}$. Ce qui se dessine, derrière cette opposition entre apaidia et eupaidia, c'est l'«idéologie civique de la maternitée ${ }^{31}$ ». Dans sa célèbre diatribe contre les femmes, Hippolyte dit rêver d'une reproduction humaine qui puisse se passer d'elles :

ô Zeus, pourquoi donc as-tu infligé aux humains ce frauduleux fléau, les femmes, en l'établissant à la lumière du soleil ? Si tu voulais propager la race mortelle, ce n'est pas aux femmes qu'il fallait en demander le moyen : contre de l'or, du fer, ou un poids de bronze déposé dans tes temples, les mortels devraient acheter de la semence d'enfants, chacun suivant la valeur du don offert ${ }^{32}$.

18 L'excès misogyne de ce discours dit toute la difficulté que rencontrent les andres, les hommes de la cité, dans leur approche de la maternité, à la fois inquiétante parce qu'échappant en partie à leur emprise et pourtant indispensable à la reproduction du groupe. C'est pour surmonter cette difficulté que se met en place, dans la société athénienne, une véritable «idéologie civique de la maternité ». La communauté des 
andres canalise à son profit la maternité en faisant d'elle la seule voie possible vers l'épanouissement de la femme grecque. C'est en deux temps que la femme acquiert sa pleine identité sociale. Le mariage légitime, qui permet à la parthénos de gagner le statut d'épouse, constitue une condition certes nécessaire, mais non suffisante à faire d'elle une femme accomplie. Ce n'est que la maternité qui lui permettra de devenir une mère eupais, le véritable archétype féminin aux yeux de la société athénienne.

La mère de douleurs

19 C'est ici que réside l'un des apports principaux de la source tragique pour une connaissance des mères grecques : elle nous renseigne moins en effet sur la maternité vécue par les femmes que sur les représentations que s'en fait la communauté masculine des citoyens athéniens. Elles sont notamment lisibles dans une série de vers qui semblent exprimer, dans l'œuvre d'Euripide, le bon sens populaire. Il s'agit de formules concises et détachables qui prennent la forme de proverbes prononcés le plus souvent par le chœur ou par son représentant, le coryphée, pour commenter l'action qui se déroule sur scène.

En ce qui concerne les mères, ces maximes à portée générale parlent essentiellement de souffrance. La séparation imminente entre Clytemnestre et sa fille Iphigénie est l'occasion pour le coryphée du chœur de jeunes femmes de Chalcis de généraliser sur la douleur des mères: "Chose étrange que la maternité : elle porte en elle un philtre puissant que nous avons toutes en partage et qui nous pousse à souffrir pour nos enfants $»^{33}$. La remarque du chef du chœur est très générale et, partant de l'exemple de Clytemnestre touchée par la douleur du deuil, elle s'étend à l'ensemble des souffrances que connaissent les mères. À en croire cette formule, la douleur est inhérente à la maternité.

21 Si certaines mères sont épargnées par la douleur du deuil, il est en tout cas une souffrance qu'elles rencontrent toutes : il s'agit de la douleur de l'enfantement, comme le rappelle une autre formule à portée générale: "C'est pour les femmes un lien étrangement fort que l'enfantement dans la douleur, et l'amour maternel est pour ainsi dire commun à tout leur sexe $»^{34}$ ! La maxime est riche d'enseignements sur ce qui fait la maternité dans l'opinion populaire grecque. La douleur de l'accouchement est à la source même de la maternité. C'est dans la souffrance que, dans un même mouvement, la femme devient mère et que l'enfant naît à la vie. L'enfantement, la douleur et la maternité fonctionnent comme un trinôme inséparable: c'est dans la douleur de l'enfantement que la maternité prend sa source; dès lors la maternité reste indissociable de la douleur par laquelle elle est née. Mais, paradoxalement, c'est précisément la douleur première de l'enfantement qui unit la mère à son enfant et qui fait du gunaikeion génos, l'« espèce " des femmes, un philoteknon génos, une "espèce » qui, par principe, par phusis, aime ses enfants.

22 Les lieux communs sur la mère que répercute le texte tragique présentent donc les mères comme des êtres de souffrances. La douleur marque le commencement de la vie maternelle et la menace de manière incessante jusqu'à la séparation d'avec l'enfant. Ce modèle d'une mère douloureuse par essence prend naissance dans les représentations masculines. Dans une société où les sentiments féminins sont rarement pris en compte, il est le signe que les douleurs de la mère sont reconnues voire valorisées, preuve encore une fois de la différence de statut qui existe entre la femme apais et la mère. Euripide est d'ailleurs allé très loin dans la valorisation des souffrances maternelles, par la voix de Médée : «On dit de nous que nous menons une vie sans péril à la maison, 
tandis qu'ils combattent à la guerre. Raisonnement insensé ! Être en ligne trois fois, le bouclier au flanc, je le préférerais à enfanter une seule ${ }^{35}$. Médée résume avec concision tout ce que l'accouchement comporte à la fois de dangers et de douleurs. Elle ose affirmer que le risque couru par les femmes en couches est supérieur à celui que courent les hoplites au combat ${ }^{36}$. Loin de laisser l'accouchement dans l'indifférence du commun, du biologique et du privé, Euripide souligne combien cet acte de la vie des femmes est un exploit au même titre que l'est, pour l'homme, la guerre.

Que ce soit dans la bouche des mères ou dans les lieux communs que prononcent les observateurs du drame, les raccourcis entre la souffrance physique de l'accouchement et la souffrance morale de la perte de l'enfant sont fréquents dans le texte tragique. Car tout passe par le corps chez la mère : la souffrance morale du deuil de l'enfant a des conséquences physiques sur le corps maternel qui semble vieillir précocement. Quand il ressent la violente douleur de la perte de l'enfant, le «corps-mémoire » de la mère ${ }^{37}$ l'associe immédiatement à la douleur première de l'enfantement.

La souffrance déborde de la psychè pour envahir le corps.

Rappelons le chœur de Créuse :

Le chœur. - C'en est fait, maîtresse, tenir un enfant dans tes bras, lui donner le sein, toi... jamais!

Ces seins et ces bras, ce sont les sièges heureux ou malheureux de la maternité. Et, dans la conception dialoguée de celle-ci, arracher de soi le nourrisson qu'on expose, cela s'exprime par le détachement physique (761-2) :

Créuse. - Ah! Si tu l'avais vu qui me tendait les bras!

Le vieillard. - Que cherchait-il ? Le sein? Tes bras pour y dormir !

Si les seins et les bras de la mère pleurent l'absence, l'enfant aussi ressent tout physiquement cette vacuité : quand Ion rêve d'un autre passé, il rêve de bras (1375-79) : Ion. - Dans le temps où j'aurais dû, choyé aux bras de ma mère, m'ouvrir à la joie de vivre, je fus privé des soins maternels tendrement nourriciers.

Et l'harmonie finale passe aussi par le corps.

De la mère à la fille : de l'identique à l'inverse

Ce ne sont pas seulement les hommes qui font le malheur des femmes, mais les femmes aussi. La question de savoir comment on devient mère illustre cet apparent paradoxe. Dans cette transformation qui est l'acquisition d'un rôle nouveau, mystérieux, à la fois désiré et redouté, les femmes sont là avant les hommes. C'est la mère qui imprime l'image du modèle social, elle qui enseigne à la vierge fille (parthénos) ses attitudes, qui l'initie aux tâches féminines qui l'aide à s'installer dans sa position de femme (gynè), épouse et mère à son tour. Ce déplacement dans l'ordre des générations passe par la sexualité, mais aussi par le changement d'identité et la transmission des rôles. La fille dépendra de la mère dont elle sera le reflet identique ou l'image inversée.

L'impossible transmission

Clytemnestre, reine d'Argos, est d'abord victime de son mari Agamemnon: «Tu m'as épousée malgré moi, tu m'as prise de force après avoir tué mon premier mari Tantale. Et le nourrisson qui se serrait sur mon sein, tu l'en as arraché pour fracasser sur le sol sa frêle vie $»^{38}$. Malgré cela, elle sera une épouse exemplaire, «J'étais pour toi, pour ta maison, une femme irréprochable $»^{39}$. "Je t'ai donné trois filles [Électre, Iphigénie, Chrysothémis] et puis ce benjamin [Oreste] et tu oses le crime de m'en arracher une ! Si 
l'on te demande pourquoi tu la mets à mort, dis, que répondras-tu? ${ }^{40}$, dit-elle à propos d'Iphigénie. Ayant été contrainte par Agamemnon d'accepter son sacrifice, elle succombe aux avances d'Égisthe et devient l'archétype de la séductrice, la mèreamante, l'incarnation de l'épouse perfide, plus $«$ femme que mère $»^{41}$. Électre, sa seconde fille, l'en accuse :

À peine ton époux avait quitté votre foyer, tu faisais devant ton miroir des arrangements de coiffure avec tes tresses blondes. Une épouse qui, quand son époux est absent de la maison, se consacre à des soins de beauté, son nom est à rayer : c'est une femme de rien. Elle n'a aucun besoin de montrer au dehors l'éclat de son plaisant visage, si elle n'est pas en quête de mal faire ${ }^{42}$.

Car non contente de s'être acheté un mari, pour lequel « elle a versé une prime pour se faire épouser ${ }^{43}$ - comportement totalement hors normes -, elle a assassiné le précédent, le roi Agamemnon de retour de sa victoire sur les Troyens accompagné de Cassandre, fille du couple royal Hécube et Priam. Dès lors, cette femme mûre éprise d'un Égisthe plus jeune rejette ses enfants «Oreste et moi nous ne sommes pour elle dans le foyer que des intrus à éliminer ${ }^{44}$; elle est l'abominable fille de Tyndaride, (la) mère (qui) l'a chassée (Électre) du palais par complaisance pour son mari $»^{45}$ mais, bien plus encore, Électre lui reproche d'être : « une femme (qui) s'attache à un mari et non ses enfants $»^{46}$. En effet, craignant une vengeance, elle exile son fils Oreste et humilie sa fille par un mariage indigne avec un paysan.

Clytemnestre l'atteste, ce n'est pas le meurtre de sa fille qui l'a incitée au geste fatal, mais la jalousie d'une épouse bafouée :

Mais Hélène était folle de son corps et de son côté le mari était incapable de punir la trahison de sa femme: voilà pourquoi il (Agamemnon) a fait périr ma fille (Iphigénie)! Là-dessus, malgré ce qu'il m'avait infligé là, je n'étais pas devenue une épouse sanguinaire, je ne l'aurais pas tué... Mais à son retour il m'est arrivé avec une fille, une possédée, une bacchante. Il l'a fourrée dans son lit! Nous allions être deux épouses installées dans son lit ! ${ }^{47}$

Avec le meurtre, débute le temps des « luttes matricides » (mètroktonous) ${ }^{48}$ entre la fille et la mère. Car Électre, est " fille-de-son-père ", le grand roi Agamemnon, comme le concède sa mère: «Tu as dans le sang une prédilection pour ton père $»^{49}$; pleure sa mort, veut le venger et arrêter là le temps de sa vie de femme. Mariée à un laboureur, apais, elle n'a jamais partagé la couche de son époux, c'est une éternelle parthénos nymphè. Une vierge qui étouffe en elle son sexe. Elle s'enlaidit, se rase la tête ${ }^{50}$, vêtue " comme une servante en corvée $»^{51}$. Ce refus des plaisirs d'Aphrodite et ses manières d'exhiber son malheur, s'ajoutent au deuil de son père; elle est «morte» avec lui ${ }^{52}$. Pour échapper à l'emprise d'une Clytemnestre toute puissante, Électre se refuse à intégrer le féminin, le temps de sa mère, celui de "l'épouse-mère " ${ }^{53}$. Plutôt que la soumission à " une mère écrasante qui la considère comme une partie d'elle-même et en dispose $\aleph^{54}$, elle choisit de se donner à un père mort et manipule son frère pour accomplir sa vengeance. Clytemnestre brouille l'ordre des générations en revivifiant son sexe, en refusant de céder la place à sa fille. En effet, c'était à Électre de prendre un époux non pas à sa mère qui ne peut plus engendrer. Ainsi rivales, la mère et la fille s'affrontent et en elles s'opposent deux générations qui auraient dû se succéder. L'une des deux doit s'effacer ou mourir.

Hélène, admirée et détestée, cette sœur de Clytemnestre donne l'image du bonheur conjugal : «Ménélas, mon bien aimé ! Long fut le temps de mon attente, mais à moi la joie aujourd'hui! Voyez amies, j'ai l'allégresse d'avoir recouvré mon époux, de le serrer 
avec amour dans mes bras après tant de matins qu'embrasa le soleil » ${ }^{55}$. Elle a une fille, Hermione, dont elle ne s'occupe guère; elles ne sont d'ailleurs jamais mises en scène ensemble, il n'y a pas de dialogue entre elles ${ }^{56}$. Toutefois lorsqu'elle fait des recommandations à sa fille pour honorer sa sœur Clytemnestre, Hélène parle comme une véritable mère, pleine de sollicitude : «Va mon enfant et quand tu auras offert les libations sur la tombe, n'oublie pas de rentrer au plus vite $»^{57}$. Elle alterne entre les deux rôles, de femme et de mère, sans les assembler. De retour de Troie, elle retrouve sa fille et "c'est près d'elle qu'Hélène trouve joie et oubli de ce qu'elle a souffert " ${ }^{58}$. Comme si celle-ci devenait la mère de sa mère. Il y a là une inversion des rôles, Hélène "glisse en position de fille $»^{59}$ pour se faire réparer par elle du dommage qu'elle a subi, la mort de sa mère qu'elle se reproche. Mais pouvait-elle devenir une «bonne » mère alors qu'elle se sent coupable de la mort de la sienne : « Ma mère a péri et son assassin, c'est moi $! »^{60}$ ? Sa fille Hermione, unique enfant qu'elle a eu de Ménélas est mariée par son père à Néoptolème. Elle est jalouse de la captive Andromaque qui a un enfant de son mari, et vit un drame dont elle accuse sa rivale d'être l'instigatrice : "Tu m'aliènes par tes philtres le cœur de mon mari et mon sein, à cause de toi se dessèche dans la stérilité » ${ }^{61}$. La clé de cette stérilité est donnée par Andromaque : "Celle qui t'a mise au monde a beaucoup aimé les hommes; n'essaie pas de la surpasser! D'une mauvaise mère, une fille se doit, si elle est raisonnable, d'éviter les façons! ${ }^{62}$ D'ailleurs Hermione le concède: "Vas-tu encore continuer à remuer tout ce qui peut me blesser? ${ }^{63}$ Comme si elle craignait de se confronter à cette mère toute-puissante " qui clôt toute possibilité de rivalité structurante, en éteignant tout espoir de l'égaler, sans même parler de la surpasser ${ }^{64}$, Hermione se maintient dans un état d'entre-deux, elle est apais, elle n'est plus la " fille-de-sa-mère » car elle a partagé la couche de son mari, mais elle ne l'a pas supplantée dans l'ordre des générations car elle ne parvient pas à porter un enfant. Pour le vieux Pelée, grand-père de Néoptolème, la chose était attendue: "Quand il s'est agi de mariage, je disais au garçon de ne pas devenir ton gendre, de ne pas prendre chez lui le produit d'une poulinière vicieuse, qui porte au dehors les hontes maternelles $»^{65}$. La mère ayant fauté, la fille ne pouvait que faillir. Victime impuissante, Hermione ne peut donner ce qu'elle n'a pas reçu. Sa peine à elle sera de ne pouvoir s'inscrire dans l'ordre de la génération. Être stérile, c'est en somme ne pas cesser d'être la fille de sa mère ${ }^{66}$.

Du mariage à la mort

Certaines mères ont vécu avec leurs filles une relation exclusive, comme Hécube avec Polyxène ou Clytemnestre avec Iphigénie. Mais vient le temps de la séparation: les filles doivent partir vers un mari ou vers la mort. C'est ainsi que les hommes séparent les filles de leurs mères. Hécube, reine d'Ilion, est une mère très aimante, presque possessive, attachée par un lien charnel à chacun de ses enfants. Polyxène est sa « fille chérie $»^{67}$. Juste avant le sacrifice de la fille, par ses gestes et ses mots, la très sage et très raisonnable reine $d$ 'Ilion devient une enfant :

N'arrache pas mon enfant de mes bras; ne me la tuez pas! Il en est mort assez. Elle est toute ma joie et l'oubli de mes maux. Pour tant de trésors perdus, c'est elle qui est mon réconfort, ma patrie, ma nourrice, l'appui et le guide de mes pas. Qui peut imposer sa loi ne doit pas l'imposer au-delà de ce qui est dû, ni s'imaginer, parce que le sort le favorise, qu'il sera toujours heureux : moi aussi je l'étais naguère, et à présent, je ne le suis plus. Tout ce dont j'étais comblée, un seul jour m'en a dépossédée ${ }^{68}$. 
fille! Touche encore ta mère... tends-moi ta main... donne... Ne me laisse pas là sans enfants! ${ }^{69}$ Devant la détresse maternelle, la fille endosse le rôle protecteur jusque-là tenu par sa mère, elle devient la mère de sa mère, pour faire le deuil de cette relation privilégiée dont la fin est proche. Polyxène répond posément et avec dignité : "Mère, écoute-moi ! (...) Pauvre maman, cesse de lutter contre qui est le maître. Veux-tu être jetée à terre, voir ton vieux corps meurtri par ceux qui te refouleront brutalement ? (...) C'est le sort qui t'attend. Non! Pas cela! Ce ne serait pas digne de nous! Donne-moi plutôt mère chérie, ta main qui m'est si douce, et ta joue à presser contre ma joue $\aleph^{70}$. Ces mots rappellent d'ailleurs ceux d'Iphigénie dans la même situation qui entend que sa mort assure « sa gloire, en chassant bien loin ce qui ne siérait qu’à un cœur mal né ! Or çà, examine avec moi, mère, combien j'ai raison. Sur moi l'Hellade immense, toute entière, a les yeux fixés. De moi dépendent le passage de la flotte et la destruction des Troyens, pour faire qu'à l'avenir les Barbares ne s'en prennent plus aux épouses, qu'ils renoncent à faire enlever des concubines dans notre Grèce bénie, quand ils auront payé cette funeste affaire $\mathrm{du}$ rapt d'Hélène par Pâris. Tous ces maux, je les écarterai $»^{71}$. Iphigénie accomplit fièrement son devoir alors que sa mère Clytemnestre implore : «Je t'accompagnerai (...) accrochée à ta robe (...). Mon enfant, tu t'en vas ? (...) Ne me quitte pas ${ }^{72}$. La séparation de la mère avec la fille est subie, violente. C'est un deuil pour la mère qui perd son enfant et son existence sociale et pour la jeune fille qui perd son statut de fille, un lendemain. Hécube se rend compte qu'elle ne sera plus ni la même mère, ni la même femme, seulement une vieille. Dès lors la mère a besoin de sa fille alors que sa fille lui échappe. C'est le deuil d'une relation exclusive, la fin d'une dépendance, toutes deux désormais seules, et sans avenir. «Prends place tout contre moi, Iphigénie : la fille sur les pas de la mère. Donne-moi ce grand bonheur, devant ces étrangères, de t'avoir debout près de moi $»^{73}$. Clytemnestre forme elle aussi un véritable couple avec sa fille ${ }^{74}$ alors que celle-ci va mourir, la reine d'Argos s'évertue à parler à la première personne du pluriel : « Si toi tu prends sur toi de me protéger de ton bras, c'est le salut pour nous. Sans cela, point de salut pour nous! $»^{75}$ On assiste à une confusion des rôles et des identités. La fille échappe à sa mère, mais se dérobe aussi à son destin de femme, au mariage et à la maternité. La mort la délivre en somme. Hécube n'est plus rien, ni femme, ni épouse, ni mère, "Jamais plus tu ne m'auras, jamais plus, en ton vieil âge, pour unir dans l'esclavage ma souffrance à ta souffrance $»^{76}$. Clytemnestre en perdant sa fille préférée va cesser d'être une mère pour les enfants qui lui restent.

Conduire sa fille au mariage est une rupture, une séparation douloureuse. Pour Clytemnestre c'est faire «don de son bien le plus cher ${ }^{77}$. Comme dans la mort, s'installe alors le deuil d'une relation : «Ne me crois pas si incapable de te comprendre, et crois bien que je souffrirai moi aussi, comme toi (...) quand je conduirai ma fille au milieu des chants nuptiaux. Mais c'est la loi de la vie, qui avec le temps tarira ces chagrins $»^{78}$. Pour une mère, donner sa fille à un époux, "c'est la loi de la vie ", c'est la perdre. D'ailleurs, dans la langue, la confusion s'entretient entre mariage et sacrifice. Écoutons les mots d'Iphigénie quand elle se décrit, partant pour Aulis, afin de s'unir « au prince des morts et non pas à Achille, cet époux dont tu m'as leurrée pour me mettre dans le char par lequel tu m'as conduite à ces noces de sang ${ }^{79}$, parée comme une mariée le jour de ses noces (« Sous le voile léger, je masquais mon visage ») ${ }^{80}$. 
souhaite marier seul sa fille : «Quoi? À l'écart d'une mère? Allez-vous me remplacer dans l'exercice d'un de mes devoirs? $\|^{81}$, car « il est de ma dignité de mère de marier moi-même mes enfants $»^{82}$, comme c'est à elle de préparer sa fille au mariage, à elle de la mener au bûcher. Le mariage est le passage obligé pour les filles, attendu pour certaines, redouté par d'autres, comme Cassandre : «Mère, à toi de me conduire (aux épousailles); et si tu vois en moi quelque répugnance, entraîne-m'y de force $»^{83}$. De cet instant qui fait de leur fille une femme, les mères sont exclues. D'ailleurs que savent ces filles de ce qui les attend? Que leur a dit leur mère ? Sans doute rien. Les supputations vont bon train, rapportées par Andromaque : «On prétend qu'il suffit d'une seule nuit à un homme pour désarmer la répugnance d'une femme devant ses étreintes ${ }^{84}$. Si l'homme les a séparées, c'est l'enfant (de préférence un fils) qui va réunir de nouveau la mère et la fille. Électre exilée ne choisit pas par hasard l'argument de l'accouchement, pour faire venir sa mère à elle : « Annonce lui que je suis accouchée d'un fils (...). Elle viendra, quand elle saura que j'ai souffert de couches ${ }^{85}$. La venue au monde de l'enfant suscite le rapprochement, il s'agit de transmettre «la part de la mère » et de redéfinir pour chacune sa place dans la génération, l'une appartenant au passé, l'autre porteuse d'avenir. C'est aussi le moment des regrets : « Je te pardonne ma petite fille. Au reste, je n'ai pas tellement à me féliciter de ma conduite, pauvre femme que je suis! Quelles déterminations j'ai prises! Je n'aurais pas dû aller si loin dans l'emportement contre mon mari $»^{86}$. Désormais mère, Électre est l'égale de Clytemnestre, pour qui le temps de l'affrontement est révolu: « Ne ravives (pas) encore la braise de nouvelles querelles ${ }^{87}$. Dorénavant, elles sont quittes, comme si les filles devaient verser le tribut d'une nouvelle vie pour prendre la place de leur mère.

Les relations d'une mère à sa fille dans le théâtre tragique sont à la fois fusionnelles et tourmentées. D'abord "miroir de sa mère $»^{88}$, la fille devra faire l'apprentissage du féminin, qui passe par le corps. Et celle qui l'a mise au monde va devoir céder la place. La séparation est douloureuse, seule l'expérience du mariage, de la mort, de la naissance permettront à la fille de devenir femme. Au centre de la relation mère-fille, il semble y avoir comme une dette $e^{89}$ qui se transmet : l'enfant que l'on doit donner à son époux, à sa cité, à sa mère aussi. Électre prétend avoir donné naissance dans le dessein de faire venir sa mère et Polyxène ou Iphigénie sur le point d'être sacrifiées regrettent amèrement ... l'hymen! Cette conception du féminin qui passe par la maternité est sans aucun doute une construction par les hommes et pour les hommes; ainsi resterontelles toutes « filles de leur mère $»^{90}$.

La tragédie est pleine de «mères en deuil ». De combien s'en faut-il pour qu'il en aille de même hors de la fiction? On ne saurait répondre. Notre connaissance, toute relative, de la culture grecque, et, plus que tout sans doute, les moyens que nous a laissés le hasard de la connaître: sans aucune "confession", aucune correspondance, aucun journal, constitués essentiellement d'oeuvres littéraires de fiction, tout cela devrait nous incliner au pessimisme. Pour autant, il est un motif fondamental pour dénier à ce défaut tout caractère paralysant : c'est cette évidence que les références tragiques à la maternité sont en harmonie - bien sûr - avec les représentations mentales de l'auteur, mais aussi avec celles de ses spectateurs. C'est notre seule justification, mais c'est toute notre justification.

41 Pour l'établissement du texte grec, nous avons utilisé les volumes consacrés à Euripide de la Collection des Universités de France, dite «Budé ». Pour les traductions, nous avons 
eu recours aussi à elles (L. Méridier, L. Parmentier, H. Grégoire, F. Chapouthier, F. Jouan); nous avons aussi utilisé parfois les traductions de Marie Delcourt (Euripide.

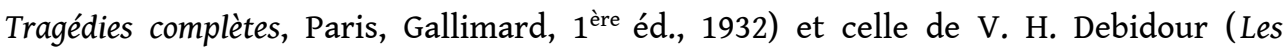
tragiques grecs. Eschyle - Sophocle - Euripide. Théâtre complet, Paris, Le Livre de Poche, 1999).

Abréviations (dans l'ordre chronologique) :

\begin{tabular}{|l|l|}
\hline Médée $:$ Med. & Hippolyte : Hipp. \\
\hline \hline Andromaque : Andr. & Hécube $:$ Hec. \\
\hline \hline Les Suppliantes : Supp. & Electre $:$ El. \\
\hline \hline Les Troyennes : Tr. & Iphigénie en Tauride : I.T. \\
\hline \hline Ion : Ion & Hélène : Hel. \\
\hline \hline Les Phéniciennes : Ph. & Oreste : Or. \\
\hline \hline Iphigénie à Aulis : I.A. & \\
\hline
\end{tabular}

\section{BIBLIOGRAPHIE}

Andre Jacques (dir.), 2003, Mères et filles. La menace de l'identique, Petite bibliothèque de psychanalyse, Paris, PUF.

Bachofen Johann Jakob, 1861, Das Mutterrecht, Stuttgart (Franckfurt am Main, 1975).

Bodiou Lydie, 2000, Histoires du sang des femmes grecques : filles, femmes, mères, d'après les écrits médicaux et biologiques, thèse de doctorat, Brulé P. (dir.), Rennes 2.

Daladier Nathalie, 1979, «Les mères aveugles », Nouvelle Revue de Psychanalyse, 19, p. 229-244.

Brulé P., 1987, La fille d'Athènes, Paris, Les Belles Lettres.

Bydlowski Monique, 1992, «Les infertiles », Nouvelle revue de psychanalyse, 45, p. 143-160.

Dupont Florence, 2001, L'insignifiance tragique, Paris, Le promeneur.

Eliacheff Caroline, Heinich Nathalie, 2003, Mères-filles. Une relation à trois, Paris, Le livre de poche. Golden Mark, 1990, Children and Childhood in Classical Athens, Baltimore-London. Héritier Françoise, cyrulnik Boris, Naouri Aldo, 1994, De l'inceste, Paris, O. Jacob. Lessana Marie-Magdeleine., 2000, Entre mère et fille : un ravage, Paris, Pluriel, Pauvert. 
Leduc Claudine, 1991, « Comment la donner en mariage ? », dans Pauline Schmitt-Pantel (éd.), Histoire des femmes, L'Antiquité, sous la dir. de G. Duby et M. Perrot, Paris, Plon, p. 259-316.

Lopez-Navarro Hélène, 2004, Les mères et les filles dans le théâtre tragique grec, Mémoire de maîtrise, Poitiers.

Loraux Nicole, 1981, « Le lit, la guerre », L'Homme, XXI, 1, p. 37-67.

Loraux Nicole, 1986, « Matrem nudam : quelques versions grecques », L'écrit du Temps, 11, p. 90-102.

Loraux Nicole, 1990, Les mères en deuil, Paris, Seuil.

Naouri Aldo, 2000, Les filles et leurs mères, O. Jacob.

Patrikiou Eleni, 1998, Structure et fonction dramatique des classes d'âge dans la société tragique d'Euripide, thèse de doctorat, Vidal-Naquet P. (dir.), EHESS, Paris.

Pierini Laurence, 2001, Mères tragiques. Les figures de la mère dans le théâtre d'Euripide, Mémoire de maîtrise, Rennes.

Ravoux-Rallo Elisabeth (dir.), 1982, La mère mauvaise, Centre d'études féminines de l'université de Provence (CEFUP), Gardanne.

Verdier Yvonne, 1979, Façons de dire, façons de faire, Paris, Gallimard.

\section{NOTES}

1. Pour les éditions et traductions utilisées, de même que pour les abréviations, voir la note bibliographique en fin d'article.

2. Ion, vers $14-15$.

3. Ce que tout un chacun, sur les bancs du théâtre, trouve tout à fait naturel. La puissance de paternité des citoyens d'Athènes est en effet telle que, dans le siècle, il est assez commun de voir des hommes se « fabriquer » des fils sans en avoir conçu, usant des fils plus ou moins surnuméraires de leurs parents ou amis.

4. On peut traduire par 'ancestral'. C'est le dieu de ces cercles de sociabilité symbolique qu'on appelle les phratries et c'est aussi le dieu de toute la cité. Les liens de parentés comme l'appartenance politique sont en quelque sorte authentifiés par Apollon Patrôos.

5. Ion, 1343.

6. Ion, 49-50.

7. En même temps que lui est révélée sa paternité par l'effet de la volonté d'Apollon, Xouthos reçoit le pouvoir de nomination, c'est lui qui nomme Ion.

8. Un fils à sa mère: Mon père a mon amour plus que personne au monde. Je le tranche tout net. Toi, n'en sois point jalouse; à lui je dois mon germe, et nul n'irait prétendre qu'il vienne de la femme : il est le fait du père (Euripide, fr. 1064).

9. Contrairement à ce qu'on a écrit, Créuse n'est pas une fille épiclère - au vrai sens, juridique, du terme. Dans le siècle, une fille n'est épiclère, c'est-à-dire en situation potentielle d'être revendiquée par un parent auprès de l'archonte, qu'à la mort de son père. Or toute la pièce illustre le fait que le roi Érechthée est toujours vivant. Il peut toujours agir sur le sort de sa fille.

10. Leduc $1991: 259-316$.

11. Ion, 298.

12. Ion, 64-65. 
13. Ion, 304-306.

14. Ion, 307-308.

15. Ion, vers 700. L'époux est alors atietos (de tiô 'honorer', 'témoigner de l'affection'), sans égard, sans tendresse.

16. Ion, 761-762.

17. Loraux 1990 : 64 .

18. Cette litanie est quasi-formulaire dans la bouche de ces femmes. Elles y ajoutent souvent le lit, la maison.

19. Hec. 810-811. Voir aussi Hec. 495-498 ; 668-669 ; Tr. 105-107 ; 1184-1185.

20. Suppl. $35 ; 168-170 ;$ Ph. 303.

21. Patrikiou $1998: 577$.

22. Il y a peu de l'apais à la ménopausée, peu de la vieille à la veuve et à la femme-quiaide d'Y. Verdier (Façons de dire, façons de faire, Paris, Gallimard, 1979), c'est-à-dire de celle qui peut impunément toucher l'intouchable (là-dessus, voir Brulé, 1987, p. 122 sq., 136 sq., 208 sq., 351-55 et 375-78).

23. Suppl., 955-959.

24. Or., 202-207

25. Comme il y a un génos des femmes, un génos « qui aime les enfants »; c'est une question de nature.

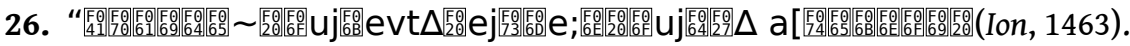

27. Ion, 490-491.

28. Ph., 1061.

29. I. A. 1386.

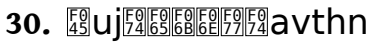

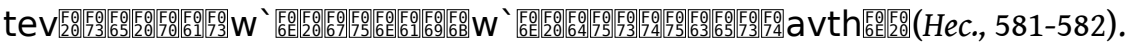

31. Loraux 1990 : 24 : « La femme n'y accomplit son télos (sa fin) qu'en enfantant et, s'il n'est pas de citoyenneté athénienne au féminin, du moins la maternité a-t-elle rang d'activité civique ».

32. Hipp., 616-623.

33. I. A., 917-918

34. Deino; $n$ gunaixi; $n$ aij di $\Delta$ wjdi;nwn gonaiv, kai; filovteknovn $p w \sim p a ` n$ gunaikei on gevno ${ }_{20}^{\circ}(P h ., 355-356)$.

35. Méd., 248-251.

36. Loraux 1981 : 44-45.

37. Loraux 1990 : 60 .

38. I. A., 1149-1151.

39. I. A., 1158-1160.

40. I. A., 1168-1170.

41. Eliacheff, Heinich, 2003 : 18. La trame de cette partie tient pour beaucoup à la lecture de ce livre Mères-filles. Une relation à trois. Ainsi qu'au travail de maîtrise d'Hélène Lopez-Navarro, Les mères et les filles dans le théâtre tragique grec, soutenu à Poitiers en juin 2004.

42. Él., 1063-1075.

43. Él., 1087.

44. Él., 62-63.

45. Él., 61-62.

46. Él., 264-265. 
47. Él., 1029-1036. C'est le plus lourd motif de la colère de Médée : qu'il en installe une autre dans son lit!

48. Tr., 363 (trad. M. Delcourt). Ce ne peut être qu'une inadvertance qui explique pourquoi L. Parmentier, dans la C.U.F., traduit par 'parricides'.

49. Él., 1103-1104. Ce qui ne manque pas d'arrière-plan idéologique : le sang de la parenté, c'est en effet celui des pères.

50. Él., 335.

51. Él., 110 et 185.

52. Loraux 1990 : $94-99$.

53. Ces propos reprennent la fine analyse de Fl. Dupont, «Électre d'Euripide, tragédie des confins », dans L'insignifiance tragique, Le promeneur, Paris, 2001, p. 135-136.

54. Ravoux-Rallo (dir.) 1982 : 93.

55. Hel., 625-629.

56. Elles sont séparées hormis dans Oreste où Hermione est une enfant.

57. Or., 125-126.

58. Or., 63.

59. Lessana $2000: 12$.

60. Hel., 208-282.

61. Andr., 158.

62. Andr., 223-226.

63. Andr., 249.

64. Eliacheff, Heinrich $2003: 88$.

65. Andr., 622.

66. Dans Oreste, Hélène meurt et Hermione se marie avec Oreste, dont elle aura un fils Tisamenos. N'ayant plus à se confronter à sa mère, Hermione se replace dans l'ordre « normal » de la génération et parvient à devenir mère : «Bien des femmes ne peuvent rencontrer l'image d'une mère vaincue qu'à travers la mort réelle de leur mère, cette mort déclenche chez nombre d'entre elles une crise à l'origine de changements : en particulier le passage de l'infertilité à la maternité », Bydlowski 1992 : 156.

67. Hec., 75.

68. $\mathrm{Hec}, 278-285$.

69. Hec., 438-441.

70. Hec., 402-414.

71. I.A., 1375-1380.

72. I.A., 1457-1470.

73. I.A., 618-620.

74. Cf. A. Naouri, «Un inceste sans passage à l'acte : la relation mère-enfant », dans F. Héritier, B. Cyrulnik, A. Naouri, De l'inceste, O. Jacob, 1994, p. 73-128. Et aussi du même auteur, Les filles et leurs mères, O. Jacob, 2000.

75. I.A., 916-917.

76. Hec., 201-204.

77. I.A., 459.

78. I.A., 690-695.

79. I.T., 368-370.

80. I. T., 371.

81. I.A., 733-734.

82. I.A., 731-732.

83. $T r ., 355$. 
84. $T r ., 664-666$.

85. Él., 655-662.

86. Él., 1099-1102.

87. Él., 1121-1122.

88. Voir André 2003 : 11-22.

89. Or., 109. « Nécessité de la dette, comme si la vie n’était pas un cadeau gratuit mais portait en soi l'exigence de transmettre ce qui a été donné et de reconnaître que ce don de vie, à la fois promesse d'immortalité et de mort, implique une dette qui circule de la mère à la fille. (...) Souvent des femmes donnent, littéralement, leur premier enfant à leur mère ", M. Bydlowski, $1992: 148$.

90. Sophocle, Électre, 365, trad. J. Irigoin, CUF, 1997.

\section{RÉSUMÉS}

C'est sur la scène tragique - dans les oeuvres d'Euripide, particulièrement -, face au public masculin de la cité, que les femmes grecques viennent crier la douleur de la maternité. Caisse de résonance de douleurs et de malheurs. De la douleur la plus féminine, celle d'enfanter, bien sûr, mais aussi du malheur le plus féminin: celui d'être apais. Certes, l'homme sans descendance souffre de cette vacuité, mais cette tare d'être sans enfant, c'est seulement pour la femme qu'elle s'étend à tout ce qui n'est pas le statut de félicité, celui de femme eupais. En effet, la femme apais, c'est aussi bien celle dont l'enfant est mort que celle à laquelle on a vite fait d'attribuer la stérilité du couple. C'est aussi celle que son âge, le délaissement dont elle est l'objet, font classer dans la catégorie des femmes vides. Coeur de la vraie vie des femmes, image fortement corporelle, la maternité constitue le pôle d'excellence, le seul vrai but de l'existence. La vierge s'y prépare à remplacer sa mère qui souffre d'abandonner ce en quoi elle s'est réalisée.

It is on the tragic stage - particularly in Euripides' dramas - in front of the male audience of the city that Greek women proclaimed their suffering in motherhood. The theater amplified their misfortune and their suffering. They wept, of course, about that most female suffering: childbirth, but also that most female misfortune : to be apais. Obviously, women shared with men the absence of children, but for women this defect of childlessness extended to all that was not the expression of happiness : to be eupais. Indeed, the apais woman included those whose child was dead, as well as the woman whom everybody held responsible for the couple's sterility. Apais women included also those whose age meant they were forsaken by men and situated within the category of empty women. Maternity was at the heart of women's lives, a strongly corporal vision of maternity, that constituted the main goal in a woman's existence. The virgin prepared then that marvelous moment when she would replace her mother who suffered to give up this stage of her life.

\section{INDEX}

Mots-clés : descendance, douleur, Euripide, fécondité, femme apais, femme eupais, génération, relation mère-fille, stérilité, tragédie 


\section{AUTEURS}

\section{LYDIE BODIOU}

Lydie BODIOU est maître de conférences d'Histoire grecque à l'Université de Poitiers, membre du laboratoire CRESCAM de Rennes. Sa thèse sur Le sang des femmes grecques (à paraître) dit assez où s'enracine son intérêt pour l'histoire des femmes et du corps. Elle a publié : « Chemins de femmes, chemins de sang en Grèce à l'époque classique ", Lunes, n² 20, juillet 2002 ; Au jardin des Hespérides. Histoire, société et épigraphie des mondes anciens, PUR, 2004.

\section{PIERRE BRULÉ}

Pierre BRULÉ est professeur d'Histoire grecque à l'Université Rennes 2 (membre du CRESCAM). Ses deux dernières publications, la direction et la rédaction du chapitre « Religion » $\mathrm{du}$ « Nouvelle Clio » IVe siècle (PUF, 2004) et Les femmes grecques à l'époque classique (Hachette, 2001, trad. angl. mod. en 2003) représentent ses deux centres d'intérêt : société et religion grecques.

\section{LAURENCE PIERINI}

Laurence PIERINI est professeur agrégée au lycée Léon Blum à Créteil (membre du CRESCAM). Après être partie à la recherche des Mères tragiques. Les figures de la mère dans le théâtre d'Euripide (2001), elle a élargi son champ d'enquête à l'oikos dans son mémoire de DEA : L'oikos et la skènè. La maison grecque dans le théâtre tragique antique (2004). 